\title{
Analyticity of Correlation Functions in One-Dimensional Classical Systems with Slowly Decreasing Potentials
}

\author{
R. L. Dobrushin \\ Institute for Problems of Information-Transmission, Moskva, USSR
}

Received February 7, 1973

\section{Introduction}

Four years ago, the following result was proved simultaneously by the author of the present paper [1-3] (for the case of general classical lattice systems), and by Ruelle [4] (for classical lattice gases). If the total energy of interaction between particles on the right-half line and particles on the left half-line is bounded, then the infinite-volume limits of the correlation functions exist, and the infinite-volume correlation functions have some rather strong cluster properties. (In the language of probability theory [5], this property is known as "uniform strong mixing" of the corresponding random processes.) The examples constructed later by Dyson [6] and by Fisher and Felderhof [7] show that the condition on the potential cannot be essentially weakened. These results have been extended to continuous systems with hard cores by the author [3] and by Gallavotti, Miracle-Sole, and Ruelle [8,9].

The above results are interpreted physically as implying the absence of phase transitions, and so it is natural to conjecture that, in these cases, thermodynamic and correlation functions vary analytically with the parameters in the interaction. For classical lattice systems with finiterange potentials such analyticity follows easily from standard theorems of linear algebra (cf. [10]), and for continuous systems with hard cores and finite-range potentials, this result was proved by van Hove [11] (see also [10]). Recently Araki proved this result for lattice systems with exponentially decreasing potentials [12]. Under similar conditions on the potential, this result can be obtained as a consequence of some results of probability theory (Statulevicius [13], Zhurbenko [14]), Gallavotti and Lin [15] proved that for potentials vanishing at infinity more rapidly than $\exp \left\{-r^{\alpha}\right\}, \alpha>0$, the thermodynamic and correlation functions are infinitely differentiable. For potentials vanishing only like an inverse 
power of $r$ previously available technique permit only the proof of the existence of finitely many derivatives (where the number of derivatives depends on the power of decrease of potential).

In this paper, the results of previous papers on the absence of phase transitions for one-dimensional systems are extended to the more general case. In particular, for potentials vanishing like an inverse power of $r$ we prove analyticity of thermodynamic and correlation functions both for lattice systems and for continuous systems with hard cores. For pair potentials, the conditions we need on the potential is that the interaction between particles on the two half lines be bounded. The same is true in the case of gases. For the general case, the conditions are to some extent more restrictive. The impossibility of an essential weakening of these conditions may be shown by examples.

To prove these results, we have developed some new techniques based on the estimation of mean values of exponential functionals of random processes. These estimates are made using new methods which can improve the results on this theme known in probability theory. The proof is not simple; we give a complete proof here only for a bounded two body potential. The proof in the general case, and some additional results, will be published elsewhere.

\section{Gibbs Distribution}

Traditionally, in the statistical physics literature, the corresponding facts about lattice and continuous systems are formulated and proved as separate results. Here we introduce a more general construction which includes both cases. Let $\mathbb{Z}=\{\ldots,-1,0,1, \ldots\}$; for any subset $V \subset \mathbb{Z}$, let $|V|$ be the number of element in $V$ and let

$$
l(V)=\min _{t \in V} t, \quad s(V)=\max _{t \in V} t, \quad d(V)=s(V)-l(V)+1 .
$$

Let $H(V), V \in \mathbb{Z}$ be the set of all subsets $C \subset \mathbb{Z}$ such that $C \cap V \neq \emptyset$ and $|C|<\infty$.

Let $X$ be some arbitrary set; we will refer to $X$ as set of states of a component. We assume $X$ is equipped with a $\sigma$-algebra $\mathfrak{B}$ of measurable subsets and a measure $m(\cdot)$ on $\mathfrak{B}$ such that $0<m(X)<\infty$. Let $X^{V}$ be the set of all mappings $y_{V}=\left(x_{t}, t \in V\right)$ where $x_{t} \in X, t \in V$. Let $\mathfrak{B}^{V}$ be the $\sigma$-algebra on $X^{V}$ which is a product of $|V|$ copies of $\mathfrak{B}$ and let $m^{V}(\cdot)$ be the product of $|V|$ copies of $m(\cdot)$ on $\mathfrak{B}^{V}$.

By a potential we mean a system of measurable functions

$$
U=\left\{U_{V}\left(y_{V}\right), V \in H(\mathbb{Z})\right\}
$$


where $y_{V} \in X^{V}$ and the values of $U_{V}$ lie in $(-\infty, \infty]$. We will consider in this paper the following conditions on the potential:

A) For any $V \in H(\mathbb{Z}), n \in \mathbb{Z}$ and $x_{t} \in X, t \in V$

$$
U_{V}\left(x_{t}, t \in V\right)=U_{V+n}\left(x_{t-n}, t \in V+n\right) .
$$

B) There exists $\mathscr{D}<\infty$ such that, for any $V \in H(\mathbb{Z}), y_{V} \in X^{V}$

$$
U_{V}\left(y_{V}\right) \geqq-\mathscr{D} .
$$

$C_{1}$ ) There exists a measurable subset $C \subset X$ with $m(C)=\mu>0$ such that ${ }^{1}$

$$
U_{V}\left(y_{V}\right) \leqq \mathscr{D} \quad \text { if } \quad x_{t_{0}} \in C \text { for some } t_{0} \in V .
$$

$\mathrm{C}_{2}$ ) There exists a measurable subset $C \subset X$ with $m(C)=\mu>0$ such that

$$
R
$$

$$
U_{V}\left(y_{V}\right)=0 \quad \text { if } \quad x_{t_{0}} \in C \text { for some } t_{0} \in V .
$$

$\mathrm{D}_{1}$ ) For some integer $L \geqq 1$

$$
\begin{aligned}
& \sum_{V \in \boldsymbol{H}((-\infty,-1]) \cap \boldsymbol{H}([0, \infty)): d(V) \geqq L} M_{U}(V) \\
& =\sum_{V \in H(\mathbb{Z}): l(V)=0, d(V) \geqq L}(d(V)-1) M_{U}(V)<\infty
\end{aligned}
$$

$\mathrm{D}_{2}$ ) For some integer $L \geqq 1$ and some $\alpha>0$

$$
\sum_{V \in \boldsymbol{H}(\mathbb{Z}), l(V)=0, d(V) \geqq L} M_{U}(V) e^{\alpha|V|}<\infty .
$$

Evidently, $C_{1}$ ) is weaker than $C_{2}$ ) and $D_{1}$ ) is weaker than $D_{2}$ ).

In the following we consider three classes of potentials:

$\mathfrak{A}$ : Those satisfying A), B), $C_{1}$ ), $D_{1}$ ).

$\mathfrak{A}_{1}$ : Those satisfying A), B), $\left.C_{1}\right), D_{2}$ ).

$\mathfrak{O}_{2}$ : Those satisfying A), B), $\left.\mathrm{C}_{2}\right), \mathrm{D}_{1}$ ).

Let

$$
\sum_{V \in H(\mathbb{Z}): l(V)=0, d(V) \geqq d}(d(V)-1) M_{U}(V) \leqq \psi(d), L \leqq d<\infty
$$

where $\psi(d)$ is a non-increasing function such that

$$
\psi(d) \rightarrow 0 \text { as } \quad d \rightarrow \infty .
$$

${ }^{1}$ Condition $C_{1}$ can be weakened. For example, for a finite-range potential and finite $X$ it is enough to require that the Markov chain corresponding to the Gibbs distribution has only one class and one subclass. A well known condition for this (see for example [16]) can easily be reformulated in terms of potential. 
We will say that conditions $\mathrm{B}$ ), $\mathrm{C}_{1}$ ), $\mathrm{D}_{1}$ ) hold uniformly in a set of potentials $\overline{\mathfrak{A}}$ if $\overline{\mathfrak{A}} \subset \mathfrak{A}$ and the constants $\mathscr{D}, \mu, L$ in these conditions and the function $\psi$ in (2.9) can be taken to be the same for all $U \in \overline{\mathfrak{A}}$.

For any $A \in H(\mathbb{Z})$ we will denote by boundary condition any function $\bar{x}=\left(x_{t}, t \in \mathbb{Z} \backslash A\right)$ where $x_{t} \in X \cup\{\theta\}, t \in \mathbb{Z} \backslash A$, and $\theta$ is called the "empty element". The equality $x_{t}=\theta$ means that there is no boundary condition at point $t$. We extend the definition of the potential by defining

$$
U_{V}\left(x_{t}, t \in V\right)=0
$$

if $x_{t_{0}}=\theta$ for some $t_{0} \in V$. The set of all such boundary conditions will be denoted by $\bar{X}(A)$. For any $\bar{x} \in \bar{X}(A)$ let $^{2}$

$$
\Phi_{A}\left(x_{t}, t \in A \mid \bar{x}\right)=\sum_{V \in H(A)} U_{V}\left(x_{t}, t \in V\right) .
$$

For any $\bar{x} \in \bar{X}(A)$ let $^{3}$

$$
F_{A}(\bar{x})=\int_{X^{A}} \exp \left\{-\Phi_{A}\left(y_{A} \mid \bar{x}\right)\right\} d y_{A} .
$$

By conditional Gibbsian distribution on $A$ with boundary condition $\bar{x} \in \bar{X}(A)$ (cf. [17]), we mean the probability distribution on $X^{A}$ defined by the following density with respect to the measure $m^{A}(\cdot)$

$$
P_{A}\left(y_{A} \mid \bar{x}\right)=\left(F_{A}(\bar{x})\right)^{-1} \exp \left\{-\Phi_{A}\left(y_{A} \mid \bar{x}\right)\right\}, y_{A} \in X^{A} .
$$

(The usual Gibbsian distribution corresponds to the boundary condition $\bar{x}_{0} \in \bar{X}(A)$ where $x_{t} \equiv \theta, t \in \mathbb{Z} \backslash A$. This will be called the trivial boundary condition.) The existence of the sums in (2.10) and the finiteness of $F_{A}(\bar{x})$ follows from conditions A), B), $\mathrm{D}_{1}$ ). The inequality

$F_{A}(\bar{x}) \geqq \mu^{|A|} \exp \left\{-\sum_{V \in H(A), d(V)<L} \mathscr{D}-\sum_{V \in H(A), d(V) \geqq L} M_{U}(V)\right\}>0$

follows from condition $\mathrm{C}_{1}$. We introduce finite dimensional densities as follows: For $B \subset A, x_{t} \in X$ for $t \in B$ and $y_{A \backslash B}=\left(x_{t}, t \in A \backslash B\right) \in X^{A \backslash B}$

$$
r_{A}^{B}\left(x_{t}, t \in B \mid \bar{x}\right)=\int_{X A B} p_{A}\left(x_{t}, t \in A \mid \bar{x}\right) d y_{A \backslash B} .
$$

Case I: Let $X$ be finite, $\mathfrak{B}$ the $\sigma$-algebra of all subsets, and $m(\cdot)$ counting measure. This is the case of classical lattice systems (see [10]). In this case $r_{A}^{B}\left(x_{t}, t \in B \mid \bar{x}\right)$ is the probability, that at each lattice site $t \in B$ there is a particle of type $x_{t}$. This case was investigated in Dobrushin [1-3] with the additional assumption that the potential is finite valued. (This is equivalent to assuming $C=X$ in $\mathrm{C}_{1}$.)

\footnotetext{
${ }^{2}$ Here and in the following we use the conventions $a+\infty=\infty$ for any $a>-\infty$ and $e^{-\infty}=0$.

${ }^{3}$ Here and in the following we will write $d y_{A}$ instead of $m^{A}\left(d y^{A}\right)$.
} 
Case II: Let $X=\{0,1, \ldots, n\} ; m, \mathfrak{B}$ as in Case I. Assume $\mathrm{C}_{2}$ ) for $C=\{0\}$. Then this is the case of a lattice gas (see [10]). When $n=1$ and $x_{t}=1, t \in B$, the functions $r_{A}^{B}$ are the usual correlation functions. Ruelle [4] and Araki [12] investigated this case with the additional assumption that $n=1$ and the potential is finite-valued.

Case III: We introduce potentials of continuous systems $U^{\prime}(\cdot)$ which are Borel functions defined on the set $H(\mathbb{R})$ of finite subsets of $\mathbb{R}$ with values in $(-\infty, \infty]$. Let $\mathfrak{U}^{\prime}$ denote the set of all potentials of continuous systems for which the following conditions $\mathrm{A}^{\prime}, \mathrm{B}^{\prime}, \mathrm{D}^{\prime}$ hold:

$\left.\mathrm{A}^{\prime}\right)$ For any $K \in H(\mathbb{R})$ and $s \in \mathbb{R}$

$$
U^{\prime}(K)=U^{\prime}(K+s) .
$$

$\left.\mathrm{B}^{\prime}\right)$ There exists $\mathscr{D}^{\prime}<\infty$ such that for any $K \in H(\mathbb{R})$

$$
U^{\prime}(K) \geqq-\mathscr{D}^{\prime} \text {. }
$$

$C^{\prime}$ ) For some $\delta>0$

$$
U^{\prime}\left(\left\{r_{1}, r_{2}\right\}\right)=\infty \quad \text { if } \quad\left|r_{1}-r_{2}\right|<\delta, r_{1} \in \mathbb{R}, r_{2} \in \mathbb{R} \backslash r_{1} .
$$

$\mathrm{D}^{\prime}$ ) For some $a, 0<a<\delta$, and $L^{\prime}>0$ which is an integral multiple of $\delta$ there exist functions $\varphi_{k}\left(v_{1}, \ldots, v_{k}\right), k=1,2, \ldots$ non-increasing in each variable, defined in domains

$$
G_{k}=\left\{v_{1} \geqq-\delta, \ldots, v_{k} \geqq-\delta, v_{1}+\cdots+v_{k} \geqq L^{\prime}-2 k \delta\right\}
$$

and such that, for any

and

$$
K=\left\{r_{1}, \ldots, r_{k+1}\right\} \in H(\mathbb{R}) \quad \text { where } \quad k \geqq 1, r_{1}<r_{2}<\cdots<r_{k+1}
$$$$
\left|U^{\prime}(K)\right| \leqq \varphi_{k}\left(r_{2}-r_{1}, \ldots, r_{k+1}-r_{k}\right) \quad \text { if } \quad\left|r_{k+1}-r_{1}\right| \geqq L^{\prime}
$$

$$
\sum_{k=1}^{\infty}\left(\frac{1}{a}\right)^{k} \int_{G_{k}} \varphi_{k}\left(v_{1}, \ldots, v_{k}\right)\left(v_{1}+\cdots+v_{k}\right) d v_{1} \ldots d v_{k}<\infty .
$$

For any Borel subset $A^{\prime} \subset \mathbb{R}$ we will define the set of boundary conditions $\bar{X}^{\prime}\left(A^{\prime}\right)$ as the set of all locally finite subsets $\bar{x}^{\prime}$ of $\mathbb{R} \backslash A^{\prime}\left(\bar{x}^{\prime}\right.$ is locally finite if its interaction with any bounded set is finite). By an evident generalization of definition (2.12) (see [3]), we introduce the notion of conditional Gibbs distribution on $A^{\prime}$ with boundary condition $\bar{x}^{\prime} \in \bar{X}^{\prime}\left(A^{\prime}\right)$. $X=[0, \delta) \cup \Lambda$ where $\Lambda$ is interpreted as the vacuum. Let $\mathfrak{B}$ be generated by the Borel subsets of $[0, \delta)$ and $\{\Lambda\}$, and let $m(\cdot)$ coincide with Lebesgue measure on $[0, \delta)$ and give $m(\{\Lambda\})=1$. Let $x_{t} \in X, t \in V, V \in H(\mathbb{Z})$

$$
U_{V}\left(x_{t}, t \in V\right)=\left\{\begin{array}{cl}
U^{\prime}\left(\left\{x_{t}+t \delta, t \in V\right\}\right) & \text { if } x_{t} \neq \Lambda \text { for all } t \in V \\
0 & \text { if } x_{t_{0}}=\Lambda \text { for some } t_{0} \in V
\end{array}\right.
$$


It is easy to see that, for such systems, there is an evident one-to-one correspondence between boundary conditions $\bar{x} \in \bar{X}(A)$ and $\bar{x}^{\prime} \in \bar{X}^{\prime}\left(A^{\prime}\right)$ where $A^{\prime}=\bigcup_{t \in A}[t \delta,(t+1) \delta$ ), partition functions (2.11) and Gibbs distributions with density (2.12) and partition functions and Gibbs distributions for the potential $U^{\prime}$. Correlation functions $h_{k}^{A^{\prime}}\left(v_{1}, \ldots, v_{k} \mid \bar{x}^{\prime}\right)$ defined in the usual way (cf. [10]) for Gibbs distributions with boundary conditions $\bar{X}^{\prime}$, where $t_{i} \delta \leqq v_{i}<\left(t_{i}+1\right) \delta, i=1, \ldots, k$, and $t_{i}$ are integers, $i=1, k$, can be expressed in terms the finite-dimensional densities as

where

$$
h_{k}^{A^{\prime}}\left(v_{1}, \ldots, v_{k} \mid \bar{x}^{\prime}\right)=r_{A}^{B}\left(x_{t}, t \in B \mid \bar{x}\right),
$$

$$
B=\left\{t_{1}, \ldots, t_{k}\right\}, \quad x_{t_{i}}=v_{l}-t_{i} \delta, \quad i=1, \ldots, k .
$$

We now show that if the potential $U^{\prime} \in \mathfrak{A}^{\prime}$ then the potential $U \in \mathfrak{A}_{2}$. It is evident that conditions $A^{\prime}$ and $B^{\prime}$ imply $A$ and $B$. Condition $C_{2}$ is true for $C=\{\Lambda\}$. From (2.7), (2.18) and (2.20) we see that, if

$$
V=\left\{0, g_{1}, g_{1}+g_{2}, \ldots, g_{1}+\cdots+g_{k}\right\}
$$

where the $g_{i}>0$ are integers, $i=1, \ldots, k, k=1,2, \ldots$ and $\delta\left(g_{1}+\cdots+g_{k}\right)$ $\geqq L^{\prime}+\delta$

$$
M_{U}(V) \leqq \varphi_{k}\left(\left(g_{1}-1\right) \delta,\left(g_{2}-1\right) \delta, \ldots,\left(g_{k}-1\right) \delta\right) .
$$

Thus, if $L=L^{\prime} \delta^{-1}+1$ and $g_{i}^{\prime}=g_{i}-1$ we find that

$$
\leqq \sum_{g_{1}^{\prime}, \ldots, g_{k}^{\prime}: g_{1}^{\prime} \geqq 0, \ldots, g_{k}^{\prime} \geqq 0, g_{1}^{\prime}+\cdots+g_{k}^{\prime} \geqq L-k-1}\left(g_{1}^{\prime}+\cdots+g_{k}^{\prime}+k\right) \varphi_{k}\left(g_{1}^{\prime} \delta, \ldots, g_{k}^{\prime} \delta\right) .
$$

Each term in the sum on the right of (2.23) can be majorized by the integral over the cube $\left\{\left(v_{1}, \ldots, v_{k}\right):\left(g_{i}^{\prime}-1\right) \delta<v_{i} \leqq g_{i}^{\prime} \delta, i=1, \ldots, k\right\}$ of the function $\delta^{-(k+1)}\left(v_{1}+v_{2}+\cdots+v_{k}+2 k \delta\right) \varphi_{k}\left(v_{1}, \ldots, v_{k}\right)$. Thus, we see from (2.23) and (2.19) that condition $D^{\prime}$ implies condition $D_{1}$.

For two-body potentials, condition $D^{\prime}$ is equivalent to the condition

$$
\left|U^{\prime}\left(\left\{r_{1}, r_{2}\right\}\right)\right| \leqq \varphi\left(\left|r_{1}-r_{2}\right|\right) \text { when }\left|r_{1}-r_{2}\right| \geqq L^{\prime}
$$

where $\varphi(v)$ is a non-increasing function such that

$$
\int_{L^{\prime}}^{\infty} \varphi(v) v d v<\infty .
$$

Such a condition was used in the paper of Gallavotti, Miracle-Sole and Ruelle [8] together with some additional assumptions related to the continuity of this function, and in the paper of Dobrushin [3] without these additional assumptions. In the paper of Gallavotti and MiracleSole [9], a condition on the decrease of the potential which is approxi- 
mately the same as our condition $\mathrm{D}^{\prime}$ is used (together with the some continuity assumptions). Conditions $\mathrm{A}^{\prime}, \mathrm{B}^{\prime}, \mathrm{C}^{\prime}, \mathrm{D}^{\prime}$ imply the conditions of Theorem 3 of Dobrushin [1] but the explicit form of the condition $\mathrm{D}^{\prime}$ was not introduced earlier.

By similar procedures it is possible to reduce to the general framework used in this paper the theory of spin systems with continuous spin (the classical Heisenberg model), gases with several species of particles, etc. It is also possibly to make a similar reduction for continuous systems of particles without hard cores and for some quantum systems (by using the representation in term of stochastic integrals), but in these cases conditions $B$ and $D_{1}$ generally do not hold.

A probability distribution for random process $\left\{\xi_{t}, t \in \mathbb{Z}\right\}$ where the random variables $\xi_{t}$ take values in $(X, \mathfrak{B})$ will be called a Gibbs state with potential $U$ if for any $A \in H(\mathbb{Z})$ the conditional distribution of $\left\{\xi_{t}, t \in A\right\}$ given $\left\{\xi_{t}, t \in \mathbb{Z} \mid A\right\}$ has the density $p_{A}\left(\cdot \mid\left\{\xi_{t}, t \in \mathbb{Z} \backslash A\right\}\right.$ ) (which was defined in (2.12)) with probability one. For the cases I, II, III considered above, this definition is the definition introduced by Dobrushin $[17,3]$ and Lanford and Ruelle [18].

\section{The Main Results}

Theorem 1. Let the potential $U \in \mathfrak{A}$. Then there exists exactly one Gibbs state with this potential. The finite-dimensional distribution of this state are given by densities $r_{B}\left(y_{B} \mid U\right), y_{B} \in X^{B}, B \in H(\mathbb{Z})$ with respect to $m^{B}(\cdot)$ such that, for any sequence of boundary conditions $\bar{x}_{n} \in \bar{X}([-n, n])$, $n=1,2, \ldots$

$$
\lim _{n \rightarrow \infty} r_{[-n, n]}^{B}\left(y_{B} \mid \bar{x}_{n}\right)=r_{B}\left(y_{B} \mid U\right)
$$

and the convergence is uniform in $y_{B} \in X^{B}$. Let $\overline{\mathfrak{A}}$ be a class of potentials on which the conditions $B, C_{1}, D_{1}$ hold uniformly. Then there exists a function $\chi(s)$ which goes to zero as $s \rightarrow \infty$ such that for all $U \in \overline{\mathfrak{A}}$, all $n \in \mathbb{N}, V \subset[-n, n]$ and any $\bar{x}_{n} \in \bar{X}([-n, n])$

$$
\begin{gathered}
\int_{X^{V}}\left|r_{V}\left(y_{V} \mid U\right)-r_{[-n, n]}^{V}\left(y_{V} \mid \bar{x}_{n}\right)\right| d y_{V} \\
\leqq \chi(n-s(V))+\chi(l(V)+n), \\
\left|r_{V}\left(y_{V} \mid U\right)-r_{[-n, n]}^{V}\left(y_{V} \mid \bar{x}_{n}\right)\right| \\
\leqq C_{V}[\chi(n-s(V))+\chi(l(V)+n)], \quad y_{V} \in X^{V},
\end{gathered}
$$

where

$$
C_{V}=\sup _{y_{V} \in X^{V}, \bar{x} \in \bar{X}(V)} p_{V}\left(y_{V} \mid \bar{x}\right)<\infty .
$$


If in (2.9), $\psi(d)=H \exp \{-h d\}, H<\infty, h>0$, it is possible to choose $\chi(s)=\hat{H} \exp \{-\hat{h} s\}, \hat{H}<\infty, \hat{h}>0$. If $\psi(d)=H d^{-h}$ it is possible to choose $\chi(s)=\hat{H} s^{-h}(\ln s)^{h+1}, \hat{H}<\infty$.

The proof of this theorem, based on some simplifications of the arguments in [1] will be published in another paper. In that paper, we obtain as a consequence of this theorem some results on the differentiability of the pressure, on cluster properties of Gibbs states, and on the existence of the limits of correlation functions for continuous systems.

Now we formulate a general theorem on the mean values of an exponential functional with respect to Gibbs states. All our results about analyticity will follow from this theorem.

Theorem 2. Let $U$ be a fixed potential in $\mathfrak{A}_{1} \cup \mathfrak{A}_{2}$; let $\psi(\bar{L})$ be a fixed function, defined for $\bar{L} \geqq L$, with $0 \leqq \psi(\bar{L})<\infty$, and with $\psi(\bar{L}) \rightarrow 0$ as $\bar{L} \rightarrow \infty$ and let $q$ be a positive number. Let $\delta>0$ and let $\mathfrak{\complement}_{\psi, q, \delta}(U)$ denote the class of all families

$$
\Gamma=\left\{\gamma_{V}\left(y_{V}\right), V \in H(\mathbb{Z})\right\}
$$

of complex-valued Borel functions of $y_{V} \in X^{V}$ having the following properties a) and b):

a) For all $V \in H(\mathbb{Z})$ and all $y_{V} \in X^{V}$

$$
\left|\gamma_{V}\left(y_{V}\right)\right| \leqq \delta\left(1+\left|U_{V}\left(y_{V}\right)\right|\right) .
$$

b) Let $C_{V}=\left\{y_{V}=\left(x_{t}, t \in V\right): x_{t_{0}} \in C\right.$ for some $\left.t_{0} \in V\right\}$ and let

$$
\begin{aligned}
& \mathscr{N}_{\Gamma}(V)=\sup _{n \in \mathbb{Z}, y_{V+n} \in X^{V+n}}\left|\gamma_{V+n}\left(y_{V+n}\right)\right|, \\
& \tilde{\mathscr{N}}_{\Gamma}(V)=\sup _{n \in \mathbb{Z}, y_{V+n} \in C_{V+n}}\left|\gamma_{V+n}\left(y_{V+n}\right)\right| .
\end{aligned}
$$

Then either $U \in \mathfrak{A}_{1}$ and

$$
\sum_{V \in \boldsymbol{H}(\mathbb{Z}), l(V)=0, d(V) \geqq \bar{L}} \mathscr{N}_{\Gamma}(V) e^{q|V|} d(V) \leqq \delta \psi(\bar{L}), \quad \bar{L} \geqq L,
$$

or $U \in \mathfrak{A}_{2}$ and

$\sum_{V \in H(\mathbb{Z}), l(V)=0, d(V) \geqq L}\left(\mathscr{N}_{\Gamma}(V)+\tilde{\mathscr{N}}_{\Gamma}(V) e^{q|V|}\right) d(V) \leqq \delta \psi(\bar{L}), \quad \bar{L} \geqq L$.

Now for any $A \in H(\mathbb{Z}), y_{A}=\left(x_{t}, t \in A\right) \in X^{A}$ and $\bar{x}=\left(x_{t}, t \in \mathbb{Z} \backslash A\right) \in \bar{X}(A)$ we define

$$
\begin{aligned}
\chi_{A}^{\Gamma}\left(y_{A} \mid \bar{x}\right) & =\sum_{V \in H(A)} \gamma_{V}\left(x_{t}, t \in V\right)^{4}, \\
g_{A}(\Gamma \mid \bar{x}) & =\int_{X^{A}} \exp \left\{-\chi_{A}^{\Gamma}\left(y_{A} \mid \bar{x}\right)\right\} p_{A}\left(y_{A} \mid \bar{x}\right) d y_{A} .
\end{aligned}
$$

\footnotetext{
${ }^{4}$ Here, as in (2.10), we put $\gamma_{V}\left(x_{t}, t \in V\right)=0$ if $x_{t_{0}}=\theta$ for some $t_{0} \in V$.
} 
Then: There exist $\delta_{0}>0$ and a function $s(\delta)$ defined for $0<\delta<\delta_{0}$ with $0 \leqq s(\delta)<1$ and with $s(\delta) \rightarrow 0$ when $\delta \rightarrow 0$ such that for all $\Gamma \in \mathfrak{C}_{\psi, q, \delta}, \delta \leqq \delta_{0}$, pairs of integers $a, b ; a \leqq b$, all $\bar{x} \in \bar{X}([a, b])$ the integral in (3.9) exists, does not vanish, and for any $\bar{x}^{\prime}=\left(\bar{x}, x_{a}^{\prime}\right) \in \bar{X}([a+1, b])$ (where $x_{a}^{\prime} \in X$ is an arbitrary element of $X$ ), we have:

$$
\left|\frac{g_{[a, b]}(\Gamma \mid \bar{x})}{g_{[a+1, b]}\left(\Gamma \mid \bar{x}^{\prime}\right)}-1\right| \leqq s(\delta)^{5} .
$$

The proof of this theorem, which is the main result of this paper, is given in Section 4 for the special case of bounded two-body potentials. The proof in the general case will be published in other papers. Theorem 2 implies the following:

Theorem 3. Let $U \in \mathfrak{A}_{1} \cup \mathfrak{A}_{2}$, a function $\psi$ and a number $q$ be fixed (as in Theorem 2), and let $\delta<\delta_{1}$ where $\delta_{1}$ is sufficiently small.

Let

$$
\Gamma(z)=\left\{\gamma_{V}\left(y_{V} ; z\right), y_{V} \in X^{V}, V \in H(\mathbb{Z})\right\} \in \mathfrak{C}_{\psi, q, \delta}(U)
$$

for all $z \in W$ where $W$ is some neighborhood of $O$ in $\mathbb{C}^{m}$, such that for each $z \in W$, each $V \in H(\mathbb{Z})$, each $n \in \mathbb{Z}$ and each $x_{t} \in X, t \in V$

$$
\gamma_{V}\left(x_{t}, t \in V ; z\right)=\gamma_{V+n}\left(\tilde{x}_{t}, t \in V+n ; z\right)
$$

where $\tilde{x}_{t+n}=x_{t}, t \in V$. Assume further that for all $V \in H(\mathbb{Z}), y_{V} \in X^{V}$ $\gamma_{V}\left(y_{V} ; z\right)$ is a holomorphic function of $z$ on $W$ vanishing at $z=0$ and having real values for $\left(z_{1}, \ldots, z_{m}\right)$ real.

For any $z \in W$ define

$$
\begin{aligned}
U_{V}\left(y_{V} ; z\right)= & U_{V}\left(y_{V}\right)+\gamma_{V}\left(y_{V} ; z\right), \quad y_{V} \in X^{V}, V \in H(\mathbb{Z}), \\
& U(z)=\left\{U_{V}\left(y_{V} ; z\right), V \in H(\mathbb{Z})\right\}
\end{aligned}
$$

and let $F_{A}(\bar{x} ; z)$ be defined by (2.10)-(2.11) with $U$ replaced by $U(z)$. Then for all pairs of integers $a \leqq b$ and all boundary conditions $\bar{x} \in \bar{X}([a, b])$ the functions

$$
f_{[a, b]}(\bar{x} ; z)=\frac{1}{b-a+1} \ln F_{[a, b]}(\bar{x} ; z)
$$

are holomorphic in $z$ on $W$ and bounded uniformly in $a, b, \bar{x}, z \in W$. For all $z \in W$ the limit

$$
f(z)=\lim _{n \rightarrow \infty} f_{[-n, n]}\left(\bar{x}_{n} ; z\right)
$$

exists, and does not depend on the sequence $\left\{\bar{x}_{n}\right\}$ of boundary conditions, and is holomorphic in $z$ on $W$.

${ }^{5}$ If $a=b$ we put $g_{[a+1, b]}\left(\Gamma \mid \bar{x}^{\prime}\right)=1$.

${ }^{6}$ Here and in the following, we consider the principal branch of the logarithm defined by the condition $\ln 1=0$. 
Proof. Theorem 3 follows easily from Theorem 2. First of all it follows easily from Eqs. (2.10)-(2.12), (3.9) and (3.13) that

$$
F_{A}(\bar{x} ; z)=F_{A}(\bar{x} ; 0) g_{A}(\Gamma(z) \mid \bar{x}) .
$$

From the definitions of $\mathfrak{U}_{1}, \mathfrak{U}_{2}$ and $\mathfrak{C}_{\psi, q, \delta}$ and Eqs. (3.9) and (2.10) it follows that there exists a finite valued function $Q(|A|)$ such that, for all $z \in W, y_{A} \in X^{A}, \bar{x} \in \bar{X}(A), A \in H(\mathbb{Z})$

$$
\left|\chi_{A}^{\Gamma(z)}\left(y_{A} \mid \bar{x}\right)\right| \leqq \delta\left(Q(|A|)+\Phi_{A}\left(y_{A} \mid \bar{x}\right)\right) .
$$

Thus, from the definition (2.12) it follows that, if $\delta_{1}<1$

$$
\int_{X^{A}}\left(\sup _{z \in W}\left|\exp \left\{-\chi_{A}^{\Gamma(z)}\left(y_{A} \mid \bar{x}\right)\right\}\right|\right) p_{A}\left(y_{A} \mid \bar{x}\right) d y_{A}<\infty .
$$

From this inequality, it follows that $g_{A}(\Gamma(z) \mid \bar{x})$ is a holomorphic function of $z \in W$. ( For example, we can use the theory of functions taking values in a Banach space ([19], 3.2.22) applied to the Banach space of bounded holomorphic functions on $W$.)

From (3.15) and (3.17) we see that, if we define $\bar{x}_{c}^{\prime} \in \bar{X}([c, b])$ (where $c$ is between $a$ and $b)$ as $\left(\bar{x}, x_{t}^{\prime}, a \leqq t<c\right)$ where the $x_{t}^{\prime}, t \in[a, b-1]$ are arbitrary (but fixed) elements of $X$ then

$$
\begin{gathered}
f_{[a, b]}(\bar{x} ; z)=f_{[a, b]}(\bar{x} ; 0) \\
+\frac{1}{b-a+1}\left[\sum_{c=a}^{b-1} \ln \frac{g_{[c, b]}\left(\Gamma(z) \mid \bar{x}_{c}^{\prime}\right)}{g_{[c+1, b]}\left(\Gamma(z) \mid \bar{x}_{c+1}^{\prime}\right)}+\ln g_{[b, b]}\left(\Gamma(z) \mid \bar{x}_{b}^{\prime}\right)\right] .
\end{gathered}
$$

If $\delta_{1}<\delta_{0}$ it follows from Eqs. (3.20) and (3.10) that the functions $f_{[a, b]}(\bar{x} ; z)$ are holomorphic and uniformly bounded functions of $z \in W$. For $\delta_{1}<1$ and for real $z$ the limit (3.16) exists as a consequence of wellknown statements about the asymptotic behaviour of partition functions. The existence of a holomorphic limit for all $z \in W$ follows, in the case $m=1$ from Vitali's Theorem about convergence of holomorphic functions. For $m \geqq 1$ we need a multidimensional generalization of Vitali's Theorem, which follows from the compactness of the space of bounded holomorphic functions (cf. [20], Theorem 1 A 12) and the theorem that a holomorphic function which vanishes for real values of its arguments is identically zero (cf. [21], p. 286).

Note that the condition of Theorem 3 is true if $m=1$ and

or if $m=1$ and

$$
\gamma_{V}\left(y_{V} ; z\right)=z U_{V}\left(y_{V}\right)
$$

$$
\gamma_{V}\left(y_{V} ; z\right)=\left\{\begin{array}{ccc}
z U_{V}\left(y_{V}\right) & \text { if } & |V|=1 \\
0 & \text { if } & |V|>1
\end{array}\right.
$$


for sufficiently small $W$. Thus, Theorem 3 implies analyticity of the pressure as a function of the inverse temperature and the chemical potential, for interactions in the class we are considering, so previous results on this question (see Section 1) are contained in Theorem 3.

In a similar way, using Theorem 2 , we can obtain results about analyticity of other physical quantities, e.g., the mean energy, and also about the analyticity of correlation functions. The above results may be reformulated as statements that physical quantities are analytic functions on Banach spaces of potentials. We will return to this point in another paper.

\section{Proof for Two-body Potentials}

Here, Theorem 2 will be proved for the special case of bounded twobody potentials and systems of function $\Gamma(z)$ of the same type, i.e. we assume

$$
\begin{array}{lll}
M_{U}(V)=0 & \text { if } & |V|>2, \\
M_{U}(V)<\infty & \text { if } & |V| \leqq 2, \\
\mathscr{N}_{\Gamma}(V)=0 & \text { if } & |V|>2, \\
\mathscr{N}_{\Gamma}(V)<\infty & \text { if } & |V| \leqq 2 .
\end{array}
$$

The main step in this proof is the following lemma:

Lemma 1. Assume the hypotheses of Theorem 2, as well as condition (4.1). Then, there exist $\delta_{0}>0$ and a function $\tilde{s}(\delta)$ defined for $0<\delta<\delta_{0}$ and tending to zero as $\delta \rightarrow 0$ such that, for all $\Gamma \in \mathfrak{C}_{\psi, q, \delta}$, integers $a \leqq b$ and $\bar{x}^{d}=\left(x_{t}^{d}, t \in \mathbb{Z} \backslash[a, b]\right) \in \bar{X}([a, b]), j=1,2$ the integral (3.9) defining the function $g_{[a, b]}\left(\Gamma \mid \bar{x}^{d}\right)$ exists, does not vanish, and satisfies

$$
\left|\frac{g_{[a, b]}\left(\Gamma \mid \bar{x}^{1}\right)}{g_{[a, b]}\left(\Gamma \mid \bar{x}^{2}\right)}-1\right| \leqq \tilde{s}(\delta) .
$$

We show that Lemma 1 implies Theorem 2. Formula (2.14) and Definition (3.9) imply that for $\bar{x}=\left(x_{t}, t \in \mathbb{Z} \backslash[a, b]\right)$

$$
\begin{aligned}
& \frac{g_{[a, b]}(\Gamma \mid \bar{x})}{g_{[a+1, b]}\left(\Gamma \mid \bar{x}^{\prime}\right)} \\
& \quad=\int_{X} \frac{g_{[a+1, b]}\left(\Gamma \mid \bar{x}\left(x_{a}\right)\right)}{g_{[a+1, b]}\left(\Gamma \mid \bar{x}^{\prime}\right)} \exp \left\{\psi\left(x_{a} \mid \bar{x}\right)\right\} r_{[a, b]}^{\{a\}}\left(x_{a} \mid \bar{x}\right) d x_{a}
\end{aligned}
$$

where $\bar{x}\left(x_{a}\right)=\left(\bar{x}, x_{a}\right) \in \bar{X}([a+1, b])$ and

$$
\psi\left(x_{a} \mid \bar{x}\right)=-\sum_{V: V \notin \boldsymbol{H}([a+1, b]), a \in V} \gamma_{V}\left(x_{t}, t \in V\right) .
$$


It now follows from (4.1), (3.5)-(3.8) that

$$
\left|\psi\left(x_{a} \mid \bar{x}\right)\right| \leqq \varphi(\delta), \quad x_{a} \in X,
$$

where $\varphi(\delta) \rightarrow 0$ as $\delta \rightarrow 0$. Theorem 2 follows from (4.2) and (4.3) by an appropriate choice of $s(\delta)$.

Lemma 2. Let $\left(W_{k}, \mathfrak{B}_{W_{k}}\right), k=1,2$, be measurable spaces, and let $V=W_{1} \times W_{2}$ be equipped with the product $\sigma$-algebra $\mathfrak{B}_{V}$. Let $m_{k}(\cdot), k=1,2$, be finite measures on $\left(W_{k}, \mathfrak{B}_{W_{k}}\right)$ and let $m(\cdot)$ be their direct product. Let $p^{i}(v), v \in V, i=1,2$, be probability densities on $\left(V, \mathfrak{B}_{V}\right)$. Let $\vec{p}^{i}\left(w_{2}\right), w_{2} \in W_{2}$ be the one dimensional densities

$$
\bar{p}^{i}\left(w_{2}\right)=\int_{W_{1}} p^{i}\left(w_{1}, w_{2}\right) m_{1}\left(d w_{1}\right), \quad i=1,2,
$$

and assume that there is a conditional density $p\left(w_{1} \mid w_{2}\right), w_{1} \in W_{1}, w_{2} \in W_{2}$ such that

$$
p^{i}\left(w_{1}, w_{2}\right)=\bar{p}^{i}\left(w_{2}\right) p\left(w_{1} \mid w_{2}\right), \quad w_{1} \in W_{1}, w_{2} \in W_{2}, i=1,2 .
$$

Let $\varphi^{1}\left(w_{2}\right), \varphi^{2}\left(w_{2}\right), w_{2} \in W_{2}$ and $\tilde{\varphi}(v), v \in V$, be measurable complex-valued functions and let $\psi^{i}(v)=\tilde{\varphi}(v) \varphi^{i}\left(w_{2}\right), v \in V, i=1,2$. Assume that, for some $\lambda$ and any two points $w \in W_{2}, w^{\prime} \in W_{2}$

$$
\left|\frac{s\left(w^{\prime}\right)}{s(w)}-1\right|<\lambda,
$$

where

$$
s(w)=\int_{W_{1}} \tilde{\varphi}\left(w_{1}, w\right) p\left(w_{1} \mid w\right) m_{1}(d w)
$$

Let

$$
\begin{aligned}
q(w) & =\min \left(\bar{p}^{1}(w), \bar{p}^{2}(w)\right), \\
\hat{p}^{1}(w) & =\bar{p}^{1}(w)-q(w), \\
\hat{p}^{2}(w) & =\bar{p}^{2}(w)-q(w), \quad w \in W_{2} .
\end{aligned}
$$

Let

$$
\varrho=\frac{1}{2} \int_{W_{2}}\left|\bar{p}^{1}(w)-\bar{p}^{2}(w)\right| m_{2}(d w)=\int_{W_{2}} \hat{p}^{i}(w) m_{2}(d w), \quad i=1,2 .
$$

and, finally, let

$$
\begin{aligned}
& \bar{g}_{i}=\int_{W_{2}}\left|\varphi^{i}(w)-1\right| \bar{p}^{i}(w) m_{2}(d w), \quad i=1,2, \\
& \hat{g}_{i}=\int_{W_{2}}\left|\varphi^{i}(w)-1\right| \hat{p}^{i}(w) m_{2}(d w), \quad i=1,2, \\
& g=\int_{W_{2}}\left|\varphi^{1}(w)-\varphi^{2}(w)\right| q(w) m_{2}(d w) .
\end{aligned}
$$


Then

$\left|\frac{\int_{V} \psi^{1}(v) p^{1}(v) m(d v)}{\int_{V} \psi^{2}(v) p^{2}(v) m(d v)}-1\right| \leqq \frac{1+\lambda}{1-(1+\lambda) \bar{g}_{2}-\lambda}\left[\lambda \varrho+g+\hat{g}_{1}+(1+\lambda) \hat{g}_{2}\right]$

provided

$$
1-(1+\lambda) \bar{g}_{2}-\lambda>0 \text {. }
$$

Proof. By using (4.6), (4.8) and (4.9), for $i=1,2$ we have:

$$
\begin{gathered}
\int_{V} \psi^{i}(v) p^{i}(v) m(d v)=\int_{W_{2}} s(w) \varphi^{i}(w) \bar{p}^{i}(w) m_{2}(d w) \\
=\int_{W_{2}} s(w) \varphi^{i}(w) q(w) m_{2}(d w)+\int_{W_{2}} s(w) \varphi^{i}(w) \hat{p}^{i}(w) m_{2}(d w) .
\end{gathered}
$$

By fixing (arbitrarily) $w^{0} \in W_{2}$ we find:

$$
\begin{aligned}
& \frac{\int_{V} \psi^{1}(v) p^{1}(v) m(d v)}{\int_{V} \psi^{2}(v) p^{2}(v) m(d v)}-1 \\
& =\left[\int_{W_{2}} \frac{s(w)}{s\left(w^{0}\right)}\left[\varphi^{1}(w)-\varphi^{2}(w)\right] q(w) m_{2}(d w)+\int_{W_{2}} \frac{s(w)}{s\left(w^{0}\right)} \varphi^{1}(w) \hat{p}^{1}(w) m_{2}(d w)\right. \\
& \left.\quad-\int_{W_{2}} \frac{s(w)}{s\left(w^{0}\right)} \varphi^{2}(w) \hat{p}^{2}(w) m_{2}(d w)\right]\left[\int_{W_{2}} \frac{s(w)}{s\left(w^{0}\right)} \varphi^{2}(w) \bar{p}_{2}(w) m_{2}(d w)\right]^{-1} .
\end{aligned}
$$

By using (4.10) we find that if $\varrho \neq 0$

$$
\begin{aligned}
& \int_{W_{2}} \frac{s(w)}{s\left(w^{0}\right)} \varphi^{1}(w) \hat{p}^{1}(w) m_{2}(d w)-\int_{W_{2}} \frac{s(\tilde{w})}{s\left(w^{0}\right)} \varphi^{2}(\tilde{w}) \hat{p}^{2}(\tilde{w}) m_{2}(d \tilde{w}) \\
& =\frac{1}{\varrho} \int_{W_{2}} \int_{W_{2}}\left[\frac{s(w)}{s\left(w^{0}\right)} \varphi^{1}(w)-\frac{s(\tilde{w})}{s\left(w^{0}\right)} \varphi^{2}(\tilde{w})\right] \hat{p}^{1}(w) \hat{p}^{2}(\tilde{w}) m_{2}(d w) m_{2}(d \tilde{w}) \\
& =\frac{1}{\varrho} \int_{W_{2}} \int_{W_{2}} \frac{s(w)}{s\left(w^{0}\right)}\left[\varphi^{1}(w)-\frac{s(\tilde{w})}{s(w)} \varphi^{2}(\tilde{w})\right] \hat{p}^{1}(w) \hat{p}^{2}(\tilde{w}) m_{2}(d w) m_{2}(d \tilde{w}) .
\end{aligned}
$$

By using first (4.7), and then (4.10), (4.12), we obtain

$$
\begin{aligned}
& \left|\int_{W_{2}} \frac{s(w)}{s\left(w^{0}\right)} \varphi^{1}(w) \hat{p}^{1}(w) m_{2}(d w)-\int_{W_{2}} \frac{s(w)}{s\left(w^{0}\right)} \varphi^{2}(w) \hat{p}^{2}(w) m_{2}(d w)\right| \\
& \leqq \frac{1+\lambda}{\varrho} \int_{W_{2}} \int_{W_{2}}\left[\left|\varphi^{1}(w)-1\right|+\left|\frac{s(\tilde{w})}{s(w)} \varphi^{2}(\tilde{w})-1\right|\right] \hat{p}^{1}(w) \hat{p}^{2}(\tilde{w}) m_{2}(d w) m_{2}(d \tilde{w}) \\
& \leqq \frac{1+\lambda}{\varrho} \int_{W_{2}} \int_{W_{2}}\left[\left|\varphi^{1}(w)-1\right|+\lambda+(1+\lambda)\left|\varphi^{2}(\tilde{w})-1\right|\right] \hat{p}^{1}(w) \hat{p}^{2}(\tilde{w}) m_{2}(d w) m_{2}(d \tilde{w}) \\
& =(1+\lambda)\left[\hat{g}_{1}+\lambda \varrho+(1+\lambda) \hat{g}_{2}\right] .
\end{aligned}
$$


(If $\varrho=0$ inequality (4.18) is trivial.) Furthermore, from (4.13) and (4.7) it follows that

$$
\left|\int_{W_{2}} \frac{s(w)}{s\left(w^{0}\right)}\left[\varphi^{1}(w)-\varphi^{2}(w)\right] q(w) m_{2}(d w)\right| \leqq(1+\lambda) g .
$$

Finally, from (4.7) and (4.11) we obtain

$$
\begin{aligned}
& \int_{W_{2}} \frac{s(w)}{s\left(w^{0}\right)} \varphi^{2}(w) \bar{p}_{2}(w) m_{2}(d w) \\
& \quad \geqq 1-\int_{W_{2}}\left|\frac{s(w)}{s\left(w^{0}\right)} \varphi^{2}(w)-1\right| \bar{p}^{2}(w) m_{2}(d w) \\
& \quad \geqq 1-\int_{W_{2}}\left(\left|\frac{s(w)}{s\left(w^{0}\right)}\right|\left|\varphi^{2}(w)-1\right|+\left|\frac{s(w)}{s\left(w^{0}\right)}-1\right|\right) \bar{p}^{2}(w) m_{2}(d w) \\
& \quad \geqq 1-(1+\lambda) \bar{g}_{2}-\lambda .
\end{aligned}
$$

Combining (4.16), (4.18), (4.19) and (4.20) gives the statement of the lemma.

Now let $\psi(\bar{L}), \bar{L} \geqq 0$, be a fixed function such that $\psi(\bar{L}) \rightarrow 0$ when $\bar{L} \rightarrow \infty$. Let $\mathfrak{Q}_{\psi}^{\prime}$ be the class of potentials $U=\left\{U_{V}\left(y_{V}\right), V \in H(\mathbb{Z})\right\}^{7}$ such that, first

and second, if

$$
M_{U}(V) \equiv 0 \text { for }|V|>2
$$

$$
\begin{aligned}
& M_{U}(d)=M_{U}(\{n, n+d\}), \quad n \in \mathbb{Z}, d=1,2, \ldots \\
& M_{U}(0)=M_{U}(\{n\}), \quad n \in \mathbb{Z}
\end{aligned}
$$

then we have

$$
\sum_{d=\bar{L}}^{\infty}(d+1) M_{U}(d) \leqq \psi(\bar{L}), \quad \bar{L}=0,1, \ldots
$$

Furthermore, let $\mathfrak{C}_{\psi, \delta}^{\prime}$ be the class of systems of functions $\Gamma$ such that, first

and second for

$$
\mathscr{N}_{\Gamma}(V) \equiv 0 \quad \text { for } \quad|V|>2
$$

$$
\begin{aligned}
& \mathscr{N}_{\Gamma}(d)=\mathscr{N}_{\Gamma}(\{n, n+d\}), \quad n \in \mathbb{Z}, d=1,2, \ldots \\
& \mathscr{N}_{\Gamma}(0)=\mathscr{N}_{\Gamma}(\{n\}), \quad n \in \mathbb{Z},
\end{aligned}
$$

then we have

$$
\sum_{d=\bar{L}}^{\infty}(d+1) \mathscr{N}_{\Gamma}(d) \leqq \delta \psi(\bar{L}), \quad \bar{L}=0,1, \ldots
$$

Since in the following we will frequently consider several potentials $U$ simultaneously, the quantities introduced in Section 2 and 3 will have the additional argument $U$. For example $g_{A}(\Gamma \mid \bar{x} ; U)$ is defined by formula (3.9) for potential $U$ and system $\Gamma$.

${ }^{7}$ We emphasize at this point that we are not assuming here that the potential is translation invariant. 
For any $A \in H(\mathbb{Z})$ and two boundary conditions

let

$$
\bar{x}^{j}=\left(x_{t}^{j}, t \in \mathbb{Z} \backslash A\right) \in \bar{X}(A), \quad j=1,2,
$$

$$
T\left(\bar{x}^{1}, \bar{x}^{2}\right)=\left\{t \in \mathbb{Z} \backslash A: x_{t}^{1} \neq x_{t}^{2}\right\} .
$$

Lemma 3. Let $\psi(\bar{L})$ be a fixed function which goes to zero as $L \rightarrow \infty$. There exist an integer $d_{0}$ and a function $\delta(c)>0(c>0)$ such that for all $c>0, A \in H(\mathbb{Z}), U \in \mathfrak{O}_{\psi}^{\prime}, \Gamma \in \mathfrak{C}_{\psi, \delta(c)}^{\prime}$ and $\bar{x}^{j}=\left(x_{t}^{j}, t \in \mathbb{Z} \backslash A\right) \in \bar{X}(A), j=1,2$, the following statements hold:

0) The integral (3.9) defining $g_{A}\left(\Gamma \mid \bar{x}^{j}, U\right)$ exists and is non-zero.

1) If one of the following four conditions holds:

a) $T\left(\bar{x}^{1}, \bar{x}^{2}\right) \subset[b, l]$ such that $\left[b-d_{0}, l\right] \subset \mathbb{Z} \backslash A$,

b) $T\left(\bar{x}^{1}, \bar{x}^{2}\right) \subset[b, l]$ such that $\left[b, l+d_{0}\right] \subset \mathbb{Z} \backslash A$,

c) $T\left(\bar{x}^{1}, \bar{x}^{2}\right) \subset(-\infty, l] \subset \mathbb{Z} \backslash A$,

d) $T\left(\bar{x}^{1}, \bar{x}^{2}\right) \subset[b, \infty) \subset \mathbb{Z} \backslash A$,

(where $b, l$ are any integers with $b \leqq l$ ) then

$$
\left|\frac{g_{A}\left(\Gamma \mid \bar{x}^{1}, U\right)}{g_{A}\left(\Gamma \mid \bar{x}^{2}, U\right)}-1\right| \leqq c,
$$

2) If $T\left(\bar{x}^{1}, \bar{x}^{2}\right) \subset[b, l] \subset \mathbb{Z} \backslash A$ with $l-b \geqq d_{0}-1$, then

$$
\left|\frac{g_{A}\left(\Gamma \mid \bar{x}^{1}, U\right)}{g_{A}\left(\Gamma \mid \bar{x}^{2}, U\right)}-1\right| \leqq 2 c .
$$

If the potential $U$ satisfies condition (4.1) and $U \in \mathfrak{A}_{1}$ then $U \in \mathfrak{O}_{\psi}^{\prime}$ for some function $\psi$. Without loss of generality, we can assume that the function $\psi$ is the same as the corresponding function in Theorem 2, so we can assume in Lemma 1 that $\Gamma \in \mathfrak{C}_{\psi, \delta}^{\prime}$. Statement (4.2) of Lemma 1 follows from inequality (4.26) (Cases c) and d)) if we take $\tilde{s}(\delta)=2 c(\delta)$ where $c(\delta)=\inf _{\delta(c) \geqq \delta} c$. The more complicated statements of Lemma 3 are needed for the proof of this lemma by induction on $|A|$.

Proof of Lemma 3. Without loss of generality, we can assume $c \leqq c_{0}$ where $c_{0}$ depends only on the function $\psi$. The function $\delta(c)$, the number $c_{0}$ and the integer $d_{0}$, which also depend only on $\psi$, are defined below in the course of the proof. Fix the set $A \in H(\mathbb{Z})$ and make the induction hypothesis that the inequalities (4.26) and (4.27) hold if $A$ is replaced by any proper subset $\tilde{A}$.

We begin by proving statement 1) of the lemma in the case where condition a) holds. (The proofs assuming b), c), or d) are similar.) By increasing $l$ if necessary, we may assume that $l+1 \in A$. Let $A=A_{0} \cup A_{1} \ldots$ $\ldots \cup A_{n}$ where each $A_{j}$ is non-empty; where $A_{0}=A \cap \Delta_{0}$ and where the 
$A_{j}, j=1, \ldots, n$, has the form $A \cap \Delta_{k_{j}}$ where the $k_{j}$ are distinct integers and

$$
\begin{aligned}
& \Delta_{k}=\left[l+d_{0} k+1, l+d_{0}(k+1)\right], \quad k=0,1, \ldots \\
& \Delta_{k}=\left[b+d_{0} k, b+d_{0}(k+1)-1\right], \quad k=-1,-2, \ldots .
\end{aligned}
$$

From condition a) and the assumption that $l+1 \in A$ it follows that such a decomposition is possible and that no $k_{j}$ is equal to -1 . We introduce the sequence of potentials $U^{j}=\left\{U_{V}^{j}\left(y_{V}\right), V \in H(\mathbb{Z})\right\}, j=0, \ldots, n+1$, by

$U_{V}^{j}\left(y_{V}\right)=\left\{\begin{array}{l}U_{V}\left(x_{s}^{2}, x_{t}\right) \text { if } V=\{s, t\} \text { where } s \in \mathbb{Z} \backslash A, t \in A_{0} \cup A_{1} \cup \cdots \cup A_{j-1} \\ U_{V}\left(y_{V}\right) \text { for other values of } V \in H(\mathbb{Z})\end{array}\right.$

(where $y_{V}=\left(x_{t}, t \in V\right) \in X^{V}$ ). Similarly we introduce the sequence of systems of functions $\Gamma^{j}=\left\{\gamma_{V}^{j}\left(y_{V}\right), V \in H(\mathbb{Z})\right\}, j=0, \ldots, n+1$, by

$\gamma_{V}^{j}\left(y_{V}\right)=\left\{\begin{array}{l}\gamma_{V}\left(x_{s}^{2}, x_{t}\right) \text { if } V=\{s, t\} \text { where } s \in \mathbb{Z} \backslash A, t \in A_{0} \cup A_{1} \cup \cdots \cup A_{j-1} \\ \gamma_{V}\left(y_{V}\right) \text { for other values of } V \in H(\mathbb{Z}) .\end{array}\right.$

Evidently $U^{0}=U, \Gamma^{0}=\Gamma$. Furthermore, from (2.10) and (3.9) it follows that

$$
\begin{gathered}
\Phi_{A}\left(y_{A} \mid \bar{x}^{2}, U\right)=\Phi_{A}\left(y_{A} \mid \bar{x}^{1}, U^{n+1}\right), \quad y_{A} \in X^{A}, \\
\chi_{A}^{\Gamma}\left(y_{A} \mid \bar{x}^{2}\right)=\chi_{A}^{\Gamma^{n+1}}\left(y_{A} \mid \bar{x}^{1}\right), \quad y_{A} \in X^{A} .
\end{gathered}
$$

Therefore, using (3.9) and (2.10)-(2.12)

so

$$
\begin{gathered}
g_{A}\left(\Gamma \mid \bar{x}^{2}, U\right)=g_{A}\left(\Gamma^{n+1} \mid \bar{x}^{1}, U^{n+1}\right) \\
\frac{g_{A}\left(\Gamma \mid \bar{x}^{1}, U\right)}{g_{A}\left(\Gamma \mid \bar{x}^{2}, U\right)}=\prod_{j=0}^{n} \frac{g_{A}\left(\Gamma^{j} \mid \bar{x}^{1}, U^{j}\right)}{g_{A}\left(\Gamma^{j+1} \mid \bar{x}^{1}, U^{j+1}\right)} .
\end{gathered}
$$

We will estimate each factor on the right of (4.32) by using Lemma 2. We let $W_{1}=X^{A \backslash A_{j}}, W_{2}=X^{A_{j}}$. The densities $p_{i}(\cdot)$ on $W_{1} \times W_{2}=X^{A}$ are defined by

$$
\begin{array}{ll}
p^{1}\left(y_{A}\right)=p_{A}\left(y_{A} \mid \bar{x}^{1}, U^{j}\right), & y_{A} \in X^{A}, \\
p^{2}\left(y_{A}\right)=p_{A}\left(y_{A} \mid \bar{x}^{1}, U^{j+1}\right), & y_{A} \in X^{A} .
\end{array}
$$

If $y_{V}=\left(x_{t}, t \in V\right) \in X^{V}, V \in H(\mathbb{Z})$, and

let

$$
\bar{x}^{1}\left(y_{A_{j}}\right)=\left(x_{t}^{1}, t \in \mathbb{Z} \backslash A ; x_{t}, t \in A_{j}\right) \in \bar{X}\left(A \backslash A_{j}\right)
$$

$$
\begin{aligned}
\varphi^{i}\left(y_{A_{j}}\right)= & \exp \left\{-\sum_{t \in A_{j}, s \in \mathbb{Z} \backslash A} \gamma_{\{s, t\}}\left(x_{s}^{i}, x_{t}\right)\right. \\
& \left.-\sum_{\{s, t\} \backslash A_{j}} \gamma_{\{s, t\}}\left(x_{s}, x_{t}\right)-\sum_{t \in A_{j}} \gamma_{\{t\}}\left(x_{t}\right),\right\} i=1,2, \\
\tilde{\varphi}\left(y_{A}\right)= & \exp \left\{-\chi_{A}^{\Gamma_{j} \backslash A_{j}}\left(y_{A \backslash A_{j}} \mid \bar{x}^{1}\left(y_{A_{j}}\right)\right\},\right. \\
\psi^{1}\left(y_{A}\right)= & \varphi^{1}\left(y_{A_{j}}\right) \tilde{\varphi}\left(y_{A}\right)=\exp \left\{-\chi_{A}^{\Gamma_{j}}\left(y_{A} \mid \bar{x}^{1}\right)\right\}, \\
\psi^{2}\left(y_{A}\right)= & \varphi^{2}\left(y_{A_{j}}\right) \tilde{\varphi}\left(y_{A}\right)=\exp \left\{-\chi_{A}^{\Gamma_{j}+1}\left(y_{A} \mid \bar{x}^{1}\right)\right\} .
\end{aligned}
$$


Then, using (3.9) we have

$$
\begin{aligned}
g_{A}\left(\Gamma^{j} \mid \bar{x}^{1}, U^{j}\right) & =\int_{X^{A}} \psi^{1}\left(y_{A}\right) p^{1}\left(y_{A}\right) d y_{A}, \\
g_{A}\left(\Gamma^{j+1} \mid \bar{x}^{1}, U^{j+1}\right) & =\int_{X^{A}} \psi^{2}\left(y_{A}\right) p^{2}\left(y_{A}\right) d y_{A} .
\end{aligned}
$$

From definitions (4.5), (4.33), and (2.14) we conclude that the marginal densities are given by:

$$
\begin{aligned}
& \bar{p}^{1}\left(y_{A_{j}}\right)=r_{A}^{A_{j}}\left(y_{A_{j}} \mid \bar{x}^{1}, U^{j}\right), \\
& \bar{p}^{2}\left(y_{A_{j}}\right)=r_{A}^{A_{J}}\left(y_{A_{j}} \mid \bar{x}^{1}, U^{j+1}\right), \quad y_{A_{j}} \in X^{A_{\jmath}} .
\end{aligned}
$$

From (4.28) and (2.10) follows that for all $y_{A \backslash A_{j}} \in X^{A \backslash A_{j}}$ and all $\bar{x} \in \bar{X}\left(A \backslash A_{j}\right)$

$$
\Phi_{A \backslash A_{j}}\left(y_{A \backslash A_{j}} \mid \bar{x}, U^{j}\right)=\Phi_{A \backslash A_{j}}\left(y_{A \backslash A_{j}} \mid \bar{x}, U^{j+1}\right)
$$

and therefore (see (4.6) and (2.14)) we obtain

$$
\begin{aligned}
p\left(y_{A \backslash A_{j}} \mid y_{A_{j}}\right) & =\frac{p_{A}\left(y_{A} \mid \bar{x}^{1}, U^{j}\right)}{r_{A}^{A_{j}}\left(y_{A_{j}} \mid \bar{x}^{1}, U^{j}\right)}=\frac{p_{A}\left(y_{A} \mid \bar{x}^{1}, U^{j+1}\right)}{r_{A}^{A_{j}}\left(y_{A_{j}} \mid \bar{x}^{1}, U^{j+1}\right)} \\
& =p_{A \backslash A_{j}}\left(y_{A \backslash A_{j}} \mid \bar{x}^{1}\left(y_{A_{j}}\right), U^{j}\right) .
\end{aligned}
$$

Thus, all the conditions of Lemma 2 are satisfied. From (4.8), (3.9), (4.34), and (4.38)

$$
s\left(y_{A_{j}}\right)=g_{A \backslash A_{j}}\left(\Gamma_{j} \mid \bar{x}^{1}\left(y_{A_{j}}\right), U^{j}\right)=g_{A \backslash A_{j}}\left(\Gamma_{j+1} \mid \bar{x}^{1}\left(y_{A_{j}}\right), U^{j+1}\right) .
$$

It follows from definitions (4.22) and (4.28) that $U^{j} \in \mathfrak{P}_{\psi}^{\prime}$ for all $j=0, \ldots$ $\ldots, n+1$. It follows similarly from definitions (4.24), (4.29) that $\Gamma^{j} \in \mathfrak{V}_{\psi, \delta}^{\prime}$ for all $j=0, \ldots, n+1$. Let $y_{A_{0}} \in X^{A_{0}}, \tilde{y}_{A_{0}} \in X^{A_{0}}$. For $A$ replaced by $A \backslash A_{0}, \bar{x}^{1}$ replaced by $\bar{x}^{1}\left(y_{A_{0}}\right), \bar{x}^{2}$ replaced by $\bar{x}^{1}\left(\tilde{y}_{A_{0}}\right)$ the hypotheses about boundary conditions in Case 1 ) of the lemma hold with $[b, l]$ replaced by $\left[b, l+d_{0}\right]$. By the induction hypothesis, in the case $j=0$

$$
\left|\frac{s\left(y_{A_{0}}\right)}{s\left(\tilde{y}_{A_{0}}\right)}-1\right| \leqq c \text {. }
$$

In a similar way, in the case $j=1, \ldots, n$, the hypotheses about boundary conditions in Case 2 ) of the lemma hold with $[b, \eta]$ replaced by $\Delta_{k}$, so by the induction hypothesis

$\left|\frac{s\left(y_{A_{j}}\right)}{s\left(\tilde{y}_{A_{j}}\right)}-1\right| \leqq 2 c, \quad y_{A_{j}} \in X^{A_{j}}, \quad \tilde{y}_{A_{j}} \in X^{A_{j}}, \quad j=1, \ldots, n$.

By comparison with (4.7) we see that we can put

$$
\lambda= \begin{cases}c & \text { if } j=0 \\ 2 c & \text { if } \quad j=1, \ldots, n .\end{cases}
$$


Using the inequality

$$
\left|e^{z}-1\right| \leqq e|z| \quad \text { if } \quad|z| \leqq 1
$$

we find, using (4.34), (4.23), and (4.24) that for $\Gamma \in \mathfrak{C}_{\psi, \delta(c)}^{\prime}$ we have $\left|\varphi^{i}\left(y_{A_{j}}\right)-1\right| \leqq 2 e d_{0} \psi(0) \delta(c), y_{A_{j}} \in X^{A_{j}}, j=0, \ldots, n, i=1,2$,

provided $\delta(c) \leqq\left(2 d_{0} \psi(0)\right)^{-1}$. (This is the first of the conditions we will impose on $\delta(c)$ in the course of the proof.) We note that, using (4.34), (4.44), (4.23), (4.43) and the fact that

we have

$$
\left\{s \in \mathbb{Z} \backslash A: x_{s}^{1} \neq x_{s}^{2}\right\} \subset[b, l]
$$

$$
\begin{aligned}
& \left|\varphi^{1}\left(y_{A_{j}}\right)-\varphi^{2}\left(y_{A_{j}}\right)\right| \\
& \quad \leqq\left|\varphi^{1}\left(y_{A_{j}}\right)\right|\left|1-\exp \left\{\sum_{t \in A_{j}, s \in \mathbb{Z} \backslash A}\left[\gamma_{\{s, t\}}\left(x_{s}^{1}, x_{t}\right)-\gamma_{\{s, t\}}\left(x_{s}^{2}, x_{t}\right)\right]\right\}\right| \\
& \quad \leqq 2 e\left(1+2 e d_{0} \psi(0) \delta(c)\right) Q_{j}
\end{aligned}
$$

where

$$
Q_{j}=\sum_{s \in[b, l], t \in A_{j}} \mathscr{N}_{\Gamma}(|t-s|) .
$$

Note also that by using (4.28), (2.10), and (4.21) we have for all $y_{A}=\left(x_{t}, t \in A\right) \in X^{A}, j=0, \ldots, n$,

where

$$
\left|\Phi_{A}\left(y_{A} \mid \bar{x}^{1}, U^{j}\right)-\Phi_{A}\left(y_{A} \mid \bar{x}^{1}, U^{j+1}\right)\right| \leqq 2 S_{j}
$$

$$
S_{j}=\sum_{s \in[b, l], t \in A_{j}} M_{U}(|t-s|) .
$$

From (2.11) and (2.12) now follows that

$$
e^{-4 S_{J}} \leqq \frac{p_{A}\left(y_{A} \mid \bar{x}^{1}, U^{j}\right)}{p_{A}\left(y_{A} \mid \bar{x}^{1}, U^{j+1}\right)} \leqq e^{4 S_{J}} .
$$

Finally, using (2.14) and (4.36), we obtain

$$
e^{-4 S_{j}} \leqq \frac{\bar{p}^{1}\left(y_{A_{j}}\right)}{\bar{p}^{2}\left(y_{A_{j}}\right)} \leqq e^{4 S_{j}}, \quad y_{A_{j}} \in X^{A_{j}}, \quad j=0, \ldots, n .
$$

From (4.10) and (4.9) it follows that

$$
\begin{aligned}
1-\varrho & =\int_{X^{A_{j}}} \min \left(\bar{p}^{1}\left(y_{A_{j}}\right), \bar{p}^{2}\left(y_{A_{j}}\right)\right) d y_{A_{j}} \\
& \geqq e^{-4 S_{J}} \int_{X^{A_{j}}} \bar{p}^{1}\left(y_{A_{j}}\right) d y_{A_{j}}=e^{-4 S_{j}}, \quad j=0, \ldots, n .
\end{aligned}
$$


The comparison of inequalities (4.44) and (4.51) with definitions (4.11), (4.12) shows that

$$
\begin{aligned}
& \bar{g}_{i} \leqq 2 e d_{0} \psi(0) \delta(c), \\
& \hat{g}_{i} \leqq 2 e d_{0} \psi(0) \delta(c)\left(1-e^{-4 S_{j}}\right) \\
& \quad \leqq 8 e d_{0} \psi(0) \delta(c) S_{j}, \quad i=1,2, \quad j=0, \ldots, n .
\end{aligned}
$$

By comparing (4.45) with (4.13) we find also

$$
g \leqq 2 e\left(1+2 e d_{0} \psi(0) \delta(c)\right) Q_{j}, \quad j=0, \ldots, n .
$$

Combining (4.35) with the statement of Lemma 2 and estimates (4.42), (4.51), (4.52), (4.53) we obtain

$$
\left|\frac{g_{A}\left(\Gamma^{j} \mid \bar{x}^{1}, U^{j}\right)}{g_{A}\left(\Gamma^{j+1} \mid \bar{x}^{1}, U^{j+1}\right)}-1\right| \leqq f_{j}, \quad j=0, \ldots, n,
$$

where (with $b_{0}=1, b_{j}=2, j=1, \ldots, n$ )

$$
\begin{aligned}
f_{j}= & \frac{1+b_{j} c}{1-\left(1+b_{j} c\right) 2 e d_{0} \psi(0) \delta(c)-b_{j} c} \\
& \cdot\left\{b_{j} c\left(1-e^{-4 S_{j}}\right)+2 e Q_{j}\left(1+2 e d_{0} \psi(0) \delta(c)\right)\right. \\
& \left.+8 e d_{0} \psi(0) \delta(c)\left(2+b_{j} c\right) S_{j}\right\}, \quad j=0, \ldots, n,
\end{aligned}
$$

provided $c$ and $\delta(c)$ are small enough so that the denominator of (4.55) is positive.

We use the following almost obvious inequality: For any complex $z_{1}, \ldots, z_{r}$ with $\left|z_{1}\right| \leqq a_{1}, \ldots,\left|z_{r}\right| \leqq a_{r}$

$$
\left|\prod_{i=1}^{r}\left(1+z_{i}\right)-1\right| \leqq \prod_{i=1}^{r}\left(1+a_{i}\right)-1 .
$$

From this, (4.32), and (4.54) follows that

$$
\left|\frac{g_{A}\left(\Gamma \mid \bar{x}^{1}, U\right)}{g_{A}\left(\Gamma \mid \bar{x}^{2}, U\right)}-1\right| \leqq \prod_{j=0}^{n}\left(1+f_{j}\right)-1 .
$$

We will choose $c_{0}$ and $\delta(c)$ such that $c_{0} \leqq(100)^{-1}$ and $\delta(c) \leqq c\left(100 d_{0} \psi(0)\right)^{-1}$. Then from (4.55) it follows that

$$
f_{j} \leqq 10\left(c S_{j}+Q_{j}\right) \text {. }
$$

Also, from (4.46), (4.24), (4.48), (4.22) and the definition of $A_{j}$ and taking into account that $k_{j} \neq 0,-1$ for $j=1, \ldots, n$ it follows that

$$
\begin{aligned}
& \sum_{j=1}^{n} Q_{j} \leqq \sum_{s \in[b, l],|t-s| \geqq d_{0}, t \notin[b, l]} \mathcal{N}_{\Gamma}(|t-s|) \leqq 2 \delta(c) \psi\left(d_{0}\right) . \\
& \sum_{j=1}^{n} S_{j} \leqq \sum_{s \in[b, l],|t-s| \geqq d_{0}, t \notin[b, l]} M_{U}(|t-s|) \leqq 2 \psi\left(d_{0}\right) .
\end{aligned}
$$


Thus it is possible to find $d_{0}$ sufficiently large (depending only on $\psi$ ) so that

$$
\prod_{j=1}^{n}\left(1+f_{j}\right)-1 \leqq \frac{c}{4} e^{-4 \psi(0)} .
$$

Fix such a value of $d_{0}$. Choose $\delta(c)$ and $c_{0}$ small enough so that (in addition to the requirements introduced above)

$$
\begin{gathered}
2 e \delta(c) \psi(0)\left(1+2 e d_{0} \psi(0) \delta(c)\right)+8 e d_{0}(\psi(0))^{2}(2+c) \delta(c) \leqq \frac{c}{2} e^{-4 \psi(0)}, \\
\frac{1+c}{1-(1+c) 2 e d_{0} \delta(c) \psi(0)-c} \leqq \frac{1-\frac{1}{3} e^{-4 \psi(0)}}{1-\frac{1}{2} e^{-4 \psi(0)}}
\end{gathered}
$$

Then by using (4.55) and the fact that (Compare (4.58)) $Q_{0} \leqq \delta(c) \psi(0)$, $S_{0} \leqq \psi(0)$ we get

$$
f_{0} \leqq\left(1-\frac{1}{3} e^{-4 \psi(0)}\right) c .
$$

By (4.59) and (4.61), and taking into account that $c \leqq c_{0} \leqq(100)^{-1}$ we have

$$
\prod_{j=0}^{n}\left(1+f_{j}\right) \leqq 1+\left(1-\frac{1}{3} e^{-4 \psi(0)}\right) c+\frac{c}{3} e^{-4 \psi(0)}=1+c .
$$

Inequalities (4.57) and (4.62) give the desired inequality (4.26).

To complete the proof of Lemma 3 , it is only necessary to check inequality (4.27) for the set $A$. The proof of inequality (4.27) is similar to the proof of (4.26), so we simply note the main changes in the argument. Let $A=A_{0} \cup A_{1} \ldots \cup A_{n}$ where each $A_{j}$ is non-empty, where $A_{0}=A \cap \Delta_{0}$, $A_{1}=A \cap \Delta_{-1}$ and where for $j=2, \ldots, n A_{j}=A \cap \Delta_{k_{j}}$ where the $\Delta_{k}$ are defined above. From the condition of Case 2) of the lemma it follows that such a partition is possible if we assume, as we may without loss of generality, that $l+1$ and $b-1$ belong to $A$. All following steps in the proof are the same, except that now the estimates for $j=1$ are the same as for $j=0$. For example, instead of (4.42), it is necessary to write

$$
\lambda=\left\{\begin{array}{ccc}
c & \text { if } & j=0,1 \\
2 c & \text { if } & j=2,3, \ldots, n .
\end{array}\right.
$$

I am very grateful to Professor Lanford for his assistance with the translation of this paper.

\section{References}

1. Dobrushin, R.L.: Description of a random field by means of conditional probabilities and regularity conditions. Probability theory and applications (in Russian) 13 (2) $201-222$ (1968). 
2. Dobrushin, R.L.: Problem of uniqueness of a Gibbs random field and phase transitions. Functional Analysis and applications (in Russian) 2 (4) 44-57 (1968).

3. Dobrushin, R.L.: Gibbs field: the general case. Functional analysis and applications (in Russian) 3 (1) 27-35 (1969).

4. Ruelle,D.: Statistical mechanics of one-dimensional lattice gas, Commun. math. Phys., 9, (4) 267-278 (1968).

5. Ibragimov, I. A., Linnik,J.V.: Independent and stationnary dependent variables (In Russian) Science edit. 1965.

6. Dyson, F.J.: Existence of a phase transitions in one-dimensional Ising Ferromagnet, Commun. math. Phys., 12, (1) 91-107 (1969).

7. Fisher, M.E., Felderhof,B.U.: Phase transition in one-dimensional cluster-interaction fluids, IA, IB, II, III, Ann. Phys., 58, (1) 176--300 (1970).

8. Gallavotti, G., Miracle-Sole,S., Ruelle,D.: Absence of phase transition in onedimensional systems with hard core, Phys. Lett., A 26, (8) 350-351 (1970).

9. Gallavotti,G., Miracle-Sole,S.: Absence of phase transitions in hard-core onedimensional systems with long-range interactions, Journ. of Math. Phys., 11, (1) 147-154 (1969).

10. Ruelle,D.: Statistical mechanics-rigorous results, N.Y.-Amsterdam: W. A. Benjamin Inc., 1969.

11. Van Hove,L.: Sur l'intégrale de configuration pour les systèmes de particules à une dimension, Physica, 16, 137-143 (1950).

12. Araki,H.: Gibbs states of one dimensional quantum lattice, Commun. math. Phys., 14, (2) 120-157, 1969.

13. Statuliavičus, V.A.: On limit theorems for stochastic functions I. Lithuanian Mathematical Collection (in Russian) 10, (3) 583-592 (1970).

14. Žurbenko,I.G.: On the determination of mixed semi-invariants for some classes of random processes (in Russian) Theor. Prob. Appl. 15 (3), 541-544 (1970).

15. Gallavotti, G.: Lin, T. F.: One dimensional lattice gases with rapidly decreasing interaction, Archive for Ration. Mech. and Analys., 37, (3) 181-191 (1970).

16. Feller, W.: An introduction to Probability Theory and its applications, vol. 1, New York: Wiley \& Sons Inc., 1950.

17. Dobrushin, R.L.: Gibbs random fields for lattice systems with pair interactions. Functional analysis and applications (in Russian) 2 (4) $31-43$ (1968).

18. Lanford,O.E., Ruelle,D.: Observables at infinity and states with short-range correlations in statistical mechanics, Commun. math. Phys., 13, (3) $194-215$ (1968).

19. Dunford, N., Schwartz, T.: Linear operators, Part 1, General Theory, New YorkLondon: Interscience Publishers, 1958.

20. Gunning, R. G., Rossi, H.: Analytic functions of several complex variables, New York: Prentice Hall, 1965.

21. Shabat, B.V.: Introduction to complex analysis (in Russian) Science edit. 1669.

\author{
R. L. Dobrushin \\ Mathematics Department \\ Chair of Probability Theory \\ Moscow State University \\ Lenin Hills \\ Moscow V 234, USSR
}


